\title{
Antibiotic use in surgical units of selected hospitals in Ghana: a multi-centre point prevalence survey
}

Antoinette A. A. Bediako-Bowan ${ }^{1,2,3,4^{*}}$ (D), Enid Owusu ${ }^{5}$, Appiah-Korang Labi ${ }^{6,7,8}$, Noah Obeng-Nkrumah ${ }^{5}$, Gifty Sunkwa-Mills ${ }^{9,10}$, Stephanie Bjerrum ${ }^{10}$, Japheth Awuletey Opintan ${ }^{9}$, Cynthia Bannerman ${ }^{11,12}$, Kåre Mølbak ${ }^{3,4}$, Jørgen Anders Lindholm Kurtzhals ${ }^{7,8}$ and Mercy Jemima Newman ${ }^{9}$

\begin{abstract}
Background: Improper use of antibiotics leads to the emergence of resistant microorganisms as well as drug toxicity, increased healthcare costs, morbidity and mortality. Globally, an estimated 25-68\% of hospitalized patients receive suboptimal antibiotic regimes. Information on the extent of this problem in Ghana is currently limited, particularly in surgical units. To strategize for interventions, we estimated the antibiotic use prevalence in surgical departments in a country-wide point prevalence survey (PPS) in Ghana.

Methods: Between October 2016 and December 2016, we conducted a cross-sectional multi-center country-wide PPS. This involved an audit of in-patients' records from all units/departments of ten systematically selected hospitals in Ghana. Data were collected with a standardized questionnaire, adopted from the European Centre for Disease Prevention and Control. In this report, we present data on antibiotic use from the surgical units.

Results: Of 2107 eligible patients included in the PPS, 540 patients were identified in surgical units, of which 70.7\% (382/540) received antibiotic therapy. A total of 636 antibiotic prescriptions were issued to these surgical patients; $224(58.6 \%)$ for treatment, including 50 for treatment of hospital-acquired infections, and 144 (37.7\%) for prophylaxis (medical and surgical). Median duration of antibiotic therapy prior to the survey was 5 days (interquartile range (IQR): 3-8 days). Surgical prophylaxis was administered for longer than the recommended one day in 107 of 144 (88.4\%) patients. The choice of antibiotics was largely similar for community- and hospital-acquired infections as well as for prophylaxis. Only 3.7\% of patients had microbiological analysis done on clinical samples.

Conclusion: We found a high prevalence of antibiotic use, with the choice of antibiotics, in some cases, inconsistent with the country's treatment guidelines. Antibiotics were administered for long duration including antibiotics for prophylactic purposes and the majority was started without supporting microbiological analysis. Prescription practices that encourage rational use of antibiotics guided by microbiology and enforcement of antibiotic policy guidelines should be the target for future interventions.
\end{abstract}

Keywords: Antibiotic use, Surgery, Surgical prophylaxis, Ghana

\footnotetext{
* Correspondence: abediako-bowan@ug.edu.gh

'Department of Surgery, School of Medicine and Dentistry, College of Health Sciences, University of Ghana, Accra, Ghana

2Department of Surgery, Korle Bu Teaching Hospital, Accra, Ghana

Full list of author information is available at the end of the article
}

(c) The Author(s). 2019 Open Access This article is distributed under the terms of the Creative Commons Attribution 4.0 International License (http://creativecommons.org/licenses/by/4.0/), which permits unrestricted use, distribution, and reproduction in any medium, provided you give appropriate credit to the original author(s) and the source, provide a link to the Creative Commons license, and indicate if changes were made. The Creative Commons Public Domain Dedication waiver (http://creativecommons.org/publicdomain/zero/1.0/) applies to the data made available in this article, unless otherwise stated. 


\section{Background}

Correct use of antimicrobials, including antibiotics, can be defined as "the cost-effective use of antimicrobials which maximizes clinical therapeutic effect, while minimizing both drug-related toxicity and the development of antimicrobial resistance" [1]. A recent systematic review unambiguously concluded that "interventions to improve antibiotic prescribing to hospital inpatients" are effective in reducing usage without increasing mortality and with resulting reduction in length-of-stay and healthcare costs [2]. However, this was almost entirely based on studies from North America and Europe and not a single study from Africa was included. Another review, focusing on low- and middle-income countries (LMIC), identified this field as a major research priority to provide tools to curb the spread of antibiotic resistance [3]. In LMIC the situation is further complicated by the fact that lack of access to relevant antibiotics can co-exist with excessive and inappropriate use [4]. Thus, research from high-income countries is of limited value to guide tailoring of antibiotic stewardship programs in African countries.

It is generally thought that inappropriate use of antimicrobials in healthcare facilities is widespread in LMIC but there are relatively few studies in limited geographical areas to document this. Generally, antimicrobial prescribing in hospitals, is said to be suboptimal in 25-68\% of cases [3, 5, 6], and in an Australian study, $47 \%$ of antibiotic use was found to be discordant with guidelines or microbiological results [7]. Some reasons ascribed to inappropriate antibiotic use include lack of regulation in antibiotic use, high rate of self-medication and lack of treatment compliance as well as lack of access to appropriate and accurate diagnostic facilities [8]. Prescribers' limited knowledge regarding optimal diagnostic approaches, lack of resources and opportunity for patient follow-up and monitoring, lack of experience and diagnostic uncertainty also lead to increasing episodes of prescribing errors $[9,10]$.

The present study aims to estimate the prevalence of antibiotic use in surgical departments at all levels of the healthcare system in Ghana and examine its variation according to patient-related and care-related characteristics. The prevalence of multidrug resistant bacteria is widespread in Ghana, higher in the regional hospitals than in the teaching hospitals [11], and a recent study has demonstrated the need for actions aimed at improving antibiotic use [12]. Most literature on antibiotic prescribing practices in surgical units in Africa focuses on surgical prophylaxis and there is a need for comprehensive data on antibiotic use in these settings. Such data are essential to design antibiotic stewardship programs, in Ghana and similar middle-income countries.

\section{Methods \\ Study design}

We analyzed a recent cross-sectional multi-center country-wide point prevalence study (PPS) of hospital acquired infections conducted at selected hospitals under the Ghana Health Service/Ministry of Health between September and December 2016 [13]. The PPS was realized through a collaboration between the selected hospitals and the HAI Ghana project research team, an international multicenter and interdisciplinary research network of Ghanaian and Danish experts in the fields of clinical microbiology, interventional studies, cost analysis and ethnographic research. Data on antibiotic use in patients admitted for a surgical specialty were analyzed for this report.

Overall in Ghana, there are 3 teaching hospitals, 10 regional hospitals and 162 district hospitals in the public health sector, with a total bed capacity of 12,806 and a $60.4 \%$ occupancy rate. District hospitals form the first referral point from health centers, with a 50-60 bed capacity and facilities for primary clinical care. Regional hospitals form the secondary level for healthcare, providing specialized care and with a 150-200 bed capacity. Tertiary hospitals provide complex tertiary care with availability of varied surgical specialties, a bed capacity ranging from 1000 to 2000 [14].

Ten representative hospitals (2 teaching hospitals, 3 regional hospitals and 5 district hospitals), one from each region, were selected across Ghana using a systematic sampling design adopted from the European Centre for Disease Prevention and Control (ECDC) methodology $[13,15]$. The total bed capacity of these 10 hospitals was 4208, representing $32.9 \%$ of the total bed capacity for the public hospitals in Ghana.

\section{Patient selection}

We included all patients on admission for a surgical specialty, irrespective of the ward they were admitted on, in the selected hospitals before $8 \mathrm{a} . \mathrm{m}$. on the day of the survey and who had not been discharged from the ward at the time of the survey. We excluded patients admitted after $8 \mathrm{a} . \mathrm{m}$., patients undergoing same day treatment or surgery; out-patients including dialysis patients; and patients in the accident and emergency departments, except for wards attached to these departments where patients are monitored for more than $24 \mathrm{~h}$.

\section{Procedure}

The survey was conducted in a single day over a 12-h period for all hospitals, except for hospitals with large bed capacity (>1000), where two or three days were used. In these large hospitals however, the survey in a designated department or unit was conducted within a 12-h period, that is 8 a.m. to 8 p.m. on a specified date. 
Five personnel at each survey site, usually members of the hospital's quality assurance team or infection control team, were recruited and trained on the data collection tool, to join the HAI Ghana research team in data collection.

Data were collected from available medical and nursing records using a modified version of the ECDC data collection tool [15]. From the records we identified patients on antibiotic therapy on the day of the survey, and recorded treatment duration for the specified agent and the results of microbiological analysis if performed. Patient-based data further included age, sex, surgical discipline, type of surgery and McCabe score [15].

\section{Statistical analysis}

Data was extracted from the multi-center country-wide study [13] and entered into ACCESS (Microsoft Office 2016) database. Data were cleaned and exported to STATA / MP version 15.1 for analysis. Statistical analysis included appropriate descriptive statistics, subpopulation estimations of percentages, interquartile range and frequencies of patients who had been administered antibiotics or not. In all calculations, the subpopulation of 540 surgical patients was analyzed as a random variable of the 2107 originally sampled patients. In addition, the two-stage sample design was taken into account by using the svyset and svy prefix of STATA. The point prevalence of antibiotic use was reported as a percentage of patients on at least one antibiotic over the total number of patients. Pearson's chi-square tests, as well as univariate and multivariate logistic regression were used to demonstrate association of various characteristics. $P$ values $<0.05$ were considered statistically significant.

\section{Results}

\section{Patient characteristics}

In total, 540 patients were admitted to surgical units in the ten selected hospitals surveyed with the following distribution by hospital category: tertiary 47\% (254), regional $18.5 \%$ (100) and district $34.4 \%$ (186). The median age was 39 years (range 3 days- 89 years [Interquartile range (IQR): 20-56 years]) and the male: female ratio was 1.7:1 Admissions to the general surgical units accounted for $71.5 \%$ (386) of patients, followed by $11.3 \%$ to the orthopedics and trauma units (Table 1). Most patients, 92\% (497), were being managed for non-fatal conditions, $6.3 \%$ (34) for ultimately fatal conditions and $1.7 \%$ (9) for rapidly fatal conditions. Of all admitted patients, 48\% (261) had undergone a surgical procedure and $11 \%$ (62) had been diagnosed with a healthcare associated infection during the current admission (Table 1).

The patients had spent a median of 8 days in hospital before the survey date, ranging from $<1$ to 203 days (IQR 4 - 18 days). For patients on antibiotic for treatment, the median duration of stay prior to the survey was 9 days (range 1 - 142 days, IQR 5-18 days) and for patients on antibiotic prophylaxis, it was 6 days (range 0 - 103 days, IQR 3-9 days].

\section{Antibiotic use}

Of all the patients, 382 (70.7\% (95\% CI 63.6-77.9\%)) received antibiotics, with an average of 1.66 prescriptions per patient. Among the 382 patients receiving antibiotics, $44.2 \%$ (169) were on one antibiotic, $46.6 \%$ (178) on two antibiotics, $7.6 \%$ (29) on three and $1.6 \%$ (6) were on four antibiotics (Additional file 1:Table S1).

Two major factors significantly affected antibiotic drug use in the univariate analysis: (1) Surgical procedure done or not $(78.6 \%$ vs $63.1 \%$, respectively, $p=0.02)$ and (2) Presence of a healthcare associated infection (HAI) (95.2\% vs 67.6 respectively, $\mathrm{p}=0.02)$. Patients admitted for a urological specialty also had a significantly increased prevalence of antibiotic use $(92.3 \%, p=0.01)$. In contrast, the hospital type, patient age, sex and disease severity were not associated with antibiotic use (Table 1) . In the final multivariable analysis, patients who had a surgical procedure, had an HAI or were managed for a urological condition, remained significantly more likely to be prescribed antibiotics (Table 1) - and these factors were independent of each other with odds ratios similar to that obtained in univariable analysis.

\section{Antimicrobial agents used}

The most frequently prescribed antibiotics across all levels of facilities were nitroimidazoles (metronidazole) (25.6\% (163 of a total of 636 prescriptions)), 2nd and 3rd generations cephalosporins (cefuroxime and ceftriaxone respectively) (20.0\% (127)), $\beta$-lactam//lactamase inhibitors (amoxicillin/clavulanic acid) (16.7\% (106)), fluoroquinolones (ciprofloxacin and levofloxacin) (12.3\% (78)) and lincosamides (clindamycin) (10.2\% (65)). The different antibiotic combinations use by groups is shown in Additional file 1: Table S1. There were some differences in the type of antibiotics prescribed between the different levels of hospitals $(p=0.002)$ : Penicillins, cephalosporins and carbapenems (meropenem) were used more in tertiary level facilities $(35.7 \%, p=0.03 ; 57.6 \%, p=0.01$ and $100 \%, p=0.01$, respectively), whereas $\beta$-lactam/ $\beta$ lactamase inhibitors were used more in district hospitals $(40.9 \%, p=0.01)$. The use of nitroimidazoles, fluoroquinolones, lincosamides and penicillins did not differ between hospital levels (Fig. 1).

The most commonly used antibiotics for treatment of both community- and hospital-acquired infections were cephalosporins, amoxicillin-clavulanic acid, clindamycin, ciprofloxacin and metronidazole (Table 2). The same antibiotics were used for surgical prophylaxis although 
Table 1 Clinical characteristics and antibiotic use pattern in surgery in hospitals in Ghana

\begin{tabular}{|c|c|c|c|c|c|c|c|}
\hline Characteristics & $\begin{array}{l}\text { Number of } \\
\text { surgical patients } \\
\text { on admissionn } \\
=540\end{array}$ & $\begin{array}{l}\text { Number of } \\
\text { surgical } \\
\text { patients on } \\
\text { antibiotics } \\
n=382\end{array}$ & $\begin{array}{l}\text { Prevalence }(95 \% \mathrm{Cl}) \\
\text { of antibiotic use } \\
\text { amongst surgical } \\
\text { patients }\end{array}$ & $\begin{array}{l}\text { Odds ratio (95\% Cl) } \\
\text { Univariable analysis }\end{array}$ & $\begin{array}{l}p^{-} \\
\text {value }\end{array}$ & $\begin{array}{l}\text { Odds ratio }(95 \% \mathrm{Cl}) \\
\text { Multivariable analysis }\end{array}$ & $\begin{array}{l}\text { Number of antibiotic } \\
\text { prescriptions } n=636 \\
\text { [Number of prescriptions } \\
\text { / persons on antibiotic] }\end{array}$ \\
\hline \multicolumn{8}{|l|}{ Hospital type } \\
\hline District Hospital & 186 & 120 & $64.5(56.0-73.1)$ & 1.0 & & & 179 [1.49] \\
\hline $\begin{array}{l}\text { Regional } \\
\text { Hospital }\end{array}$ & 100 & 84 & $84.0(68.3-99.7)$ & $2.9(0.8-9.9)$ & 0.08 & & 166 [1.98] \\
\hline Tertiary Hospital & 254 & 178 & $70.1(64.8-75.3)$ & $1.3(0.8-2.0)$ & 0.2 & & 291 [1.63] \\
\hline \multicolumn{8}{|l|}{ Surgical specialty } \\
\hline $\begin{array}{l}\text { Dental (+ MXF) } \\
\text { Surgery }\end{array}$ & 13 & 9 & $69.2(52.2-86.3)$ & 1.0 & & & $19[2.1]$ \\
\hline $\begin{array}{l}\text { Ear, Nose and } \\
\text { Throat }\end{array}$ & 8 & 7 & $87.5(50.6-124.4)$ & $3.1(0.1-95.1)$ & 0.5 & & 10 [1.43] \\
\hline General Surgery & 386 & 274 & $71.0(61.3-80.7)$ & $1.1(0.4-2.7)$ & 0.8 & & $466[1.70]$ \\
\hline Neurosurgery & 24 & 15 & $62.5(62.5-62.5)$ & $0.7(0.3-1.6)$ & 0.4 & & 26 [1.73] \\
\hline Ophthalmology & 2 & 0 & 0.0 & - & & & $0[0.00]$ \\
\hline $\begin{array}{l}\text { Orthopedics and } \\
\text { trauma }\end{array}$ & 61 & 40 & $65.6(62.1-69.1)$ & $0.8(0.4-1.9)$ & 0.7 & & 52 [1.30] \\
\hline Pediatric surgery & 33 & 25 & $75.8(67.7-83.8)$ & $1.3(0.6-3.4)$ & 0.4 & & 42 [1.68] \\
\hline Urology & 13 & 12 & $92.3(92.3-92.3)$ & $5.3(2.3-11.9)$ & 0.001 & $5.0(1.5-15.9)$ & 21 [1.75] \\
\hline \multicolumn{8}{|l|}{ Age group } \\
\hline 0-12 (Children) & 82 & 62 & $75.6(64.7-86.5)$ & 1.0 & & & 111 [1.79] \\
\hline $\begin{array}{l}\text { 13-17 } \\
\text { (Adolescents) }\end{array}$ & 33 & 21 & $63.6(41.2-86.1)$ & $0.6(0.2-1.5)$ & 0.2 & & 36 [1.71] \\
\hline $\begin{array}{l}\text { 18-29 (Young } \\
\text { adult) }\end{array}$ & 84 & 58 & $69.0(61.6-76.5)$ & $0.7(0.4-1.4)$ & 0.3 & & 100 [1.72] \\
\hline 30-39 (Thirties) & 75 & 51 & $68.0(49.6-86.4)$ & $0.7(0.4-1.3)$ & 0.2 & & 80 [1.57] \\
\hline $\begin{array}{l}\text { 40-64 (Middle } \\
\text { age) }\end{array}$ & 170 & 121 & $71.2(64.0-78.4)$ & $0.8(0.4-1.5)$ & 0.4 & & 198 [1.64] \\
\hline$>65$ (Old age) & 96 & 69 & $71.9(61.7-82.6)$ & $0.8(0.4-1.9)$ & 0.6 & & $111[1.61]$ \\
\hline \multicolumn{8}{|l|}{ Gender } \\
\hline Male & 340 & 243 & 71.5 (63.1-79.8) & 1.0 & & & 397 [1.63] \\
\hline Female & 200 & 139 & $69.5(62.1-76.9)$ & $0.9(0.6-1.3)$ & 0.6 & & 239 [1.72] \\
\hline \multicolumn{8}{|l|}{ McCabe Score } \\
\hline Non-fatal & 497 & 356 & 71.6 (63.9-79.3) & 1.0 & & & $594[1.67]$ \\
\hline Rapidly fatal & 9 & 6 & $66.7(16.7-116.6)$ & $0.8(0.1-5.8)$ & 0.8 & & 8 [1.33] \\
\hline Ultimately fatal & 34 & 20 & $58.8(45.6-72.0)$ & $0.6(0.3-1.2)$ & 0.1 & & 34 [1.70] \\
\hline \multicolumn{8}{|c|}{ Surgical procedure done } \\
\hline No & 278 & 175 & $63.1(50.9-75.3)$ & 1.0 & & & $293[1.67]$ \\
\hline Yes & 262 & 207 & $77.6(72.1-85.0)$ & $2.1(1.5-3.1)$ & 0.02 & $2.2(1.0-4.6)$ & $343[1.66]$ \\
\hline \multicolumn{8}{|l|}{ HAl documented } \\
\hline No & 478 & 323 & $67.6(59.8-75.4)$ & 1.0 & & & $522[1.62]$ \\
\hline Yes & 62 & 59 & $95.2(86.8-103.6)$ & $9.4(1.5-60.3)$ & 0.02 & $8.9(1.4-56.5)$ & 114 [1.93] \\
\hline \multicolumn{8}{|l|}{ Types of infections } \\
\hline$B S I$ & 4 & 4 & 100.0 & & & & $9[2.25]$ \\
\hline Pneumonia & 7 & 7 & 100.0 & & & & $18[2.57]$ \\
\hline SSI & 36 & 33 & $91.7(79.5-103.8)$ & & & & $61[1.85]$ \\
\hline
\end{tabular}


Table 1 Clinical characteristics and antibiotic use pattern in surgery in hospitals in Ghana (Continued)

\begin{tabular}{|c|c|c|c|c|c|c|}
\hline Characteristics & $\begin{array}{l}\text { Number of } \\
\text { surgical patients } \\
\text { on admissionn } \\
=540\end{array}$ & $\begin{array}{l}\text { Number of } \\
\text { surgical } \\
\text { patients on } \\
\text { antibiotics } \\
n=382\end{array}$ & $\begin{array}{l}\text { Prevalence }(95 \% \mathrm{Cl}) \\
\text { of antibiotic use } \\
\text { amongst surgical } \\
\text { patients }\end{array}$ & $\begin{array}{l}\text { Odds ratio }(95 \% \mathrm{Cl}) \\
\text { Univariable analysis } \\
\text { Ualue }\end{array}$ & $\begin{array}{l}\text { Odds ratio }(95 \% \mathrm{Cl}) \\
\text { Multivariable analysis }\end{array}$ & $\begin{array}{l}\text { Number of antibiotic } \\
\text { prescriptions } n=636 \\
\text { [Number of prescriptions } \\
\text { / persons on antibiotic] }\end{array}$ \\
\hline UTI & 7 & 7 & 100.0 & & & $11[1.57]$ \\
\hline Other & 8 & 8 & 100.0 & & & 15 [1.88] \\
\hline
\end{tabular}

MXF Maxillofacial, McCabe score: Classification of the severity of underlying medical conditions. Non-fatal disease (expected survival at least five years); ultimately fatal disease (expected survival between one and five years); rapidly fatal disease (expected death within one year); $\mathrm{Cl} C$ onfidence interval, $\mathrm{HAl}$ Healthcare associated infection, BSI Blood stream infection, SSI Surgical site infection, UTI Urinary tract infection

clindamycin and metronidazole were used less for this indication.

\section{Indication for antibiotic use}

Patients were prescribed antibiotics for treatment of infections $(58.6 \%(224 / 382))$ or for prophylaxis $(37.7 \%$ (144/382)), and for 14 patients there were no recorded reason for antibiotic therapy. Of patients prescribed antibiotics for treatment, $77.7 \%$ (174/224) were given for community acquired infections - including cellulitis, osteomyelitis, septicemia, urinary tract infections, pyelonephritis, intracerebral abscesses and peritonitis due to typhoid ileitis with perforation, appendix abscesses and perforated duodenal ulcers; and 22.3\% (50/224) for hospital acquired infections. Of the antibiotics used for prophylaxis, $16 \%(23 / 144)$ were given for medical

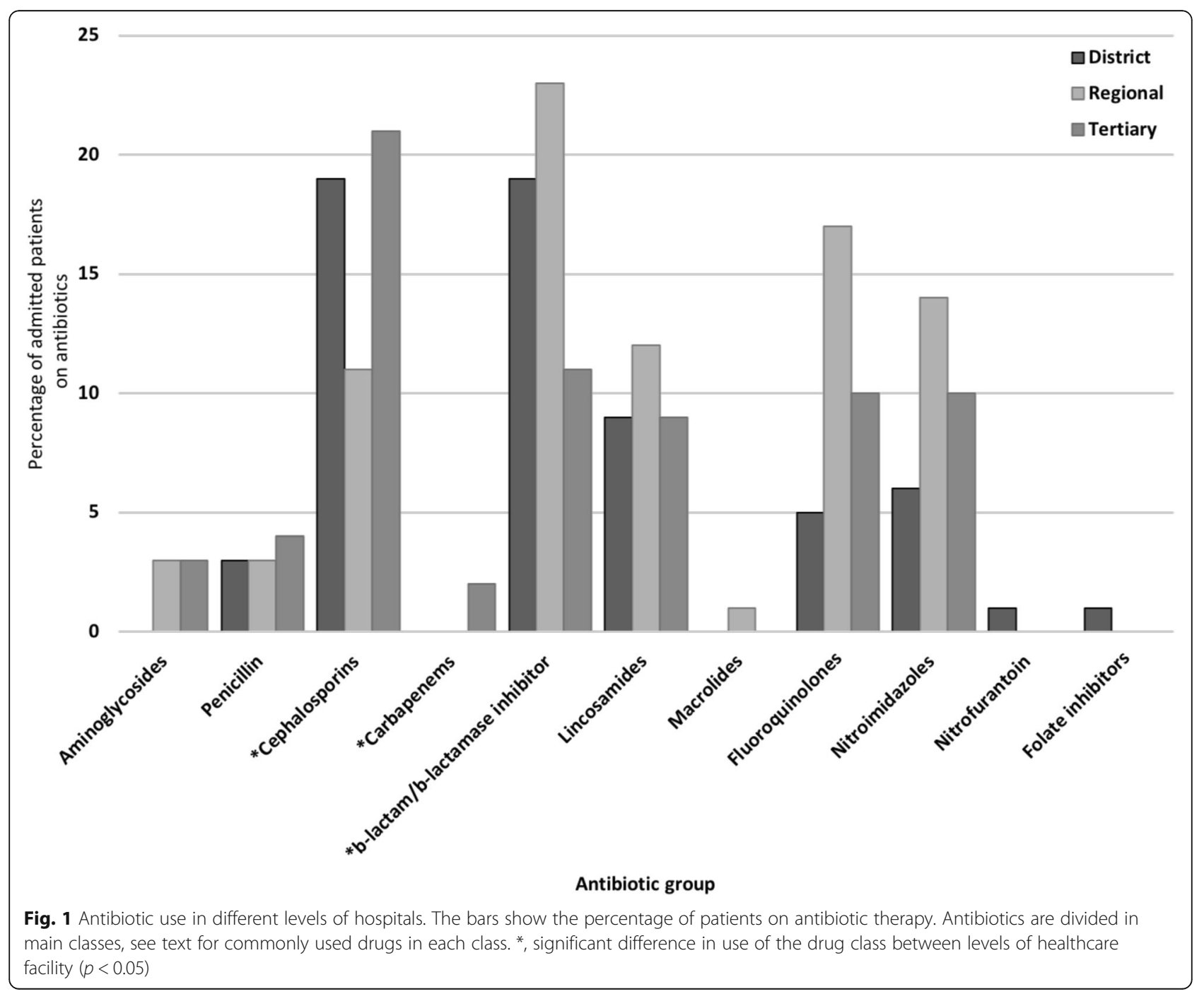


Table 2 Indication of antibiotic use in patients of selected hospitals in Ghana

\begin{tabular}{|c|c|c|c|c|c|}
\hline \multirow[b]{2}{*}{ Antibiotic group (n) } & \multicolumn{5}{|c|}{ Indication for treatment $(n=382)$} \\
\hline & $\begin{array}{l}\text { Community acquired } \\
\text { infection }(n=174)\end{array}$ & $\begin{array}{l}\text { Surgical prophylaxis } \\
(n=121)\end{array}$ & $\begin{array}{l}\text { Hospital acquired } \\
\text { infection }(n=53)\end{array}$ & $\begin{array}{l}\text { Medical prophylaxis } \\
(n=23)\end{array}$ & $\begin{array}{l}\text { Unknown indication } \\
(n=14)\end{array}$ \\
\hline Aminoglycosides (10) & 5 & 0 & 3 & 1 & 1 \\
\hline Penicillin (28) & 15 & 8 & 4 & 1 & 0 \\
\hline Cephalosporins (92) & 40 & 35 & 9 & 5 & 3 \\
\hline Carbapenems (5) & 1 & 1 & 3 & 0 & 0 \\
\hline $\begin{array}{l}\beta \text {-lactam/ } \beta \text {-lactamase } \\
\text { inhibitor (88) }\end{array}$ & 31 & 34 & 11 & 7 & 5 \\
\hline Lincosamides (51) & 34 & 8 & 6 & 1 & 2 \\
\hline Macrolides (1) & 0 & 0 & 1 & 0 & 0 \\
\hline Fluoroquinolones (53) & 18 & 23 & 8 & 3 & 1 \\
\hline Nitroimidazoles (51) & 30 & 11 & 5 & 4 & 1 \\
\hline Nitrofurantoin (2) & 0 & 1 & 0 & 0 & 1 \\
\hline Folate inhibitors (1) & 0 & 0 & 0 & 1 & 0 \\
\hline
\end{tabular}

prophylaxis and 84\% (121/144) for surgical prophylaxis. The contribution of surgical prophylaxis to the total antibiotic usage was 32\% (121 of 382 patients given antibiotics). For surgical prophylaxis, $88.4 \%(107 / 121)$ of the patients received antibiotics for more than one day, 9.9\% $(12 / 121)$ for one day and $1.6 \%(2 / 121)$ as a single dose. The distribution between antibiotic use for communityand hospital-acquired infections and prophylactic use was similar at district, regional and tertiary facilities $(p=0.7)$.

\section{Route of administration}

Antibiotics were more commonly administered per the parenteral route $(54 \%)$ than orally $(46 \%)$. For individual antibiotic agents, nitroimidazoles (metronidazole) were mainly given by the parenteral route $(67 \%)$, whereas $\beta$ lactam/ $\beta$-lactamase inhibitors (amoxicillin/clavulanic acid) were mainly given orally (53\%). All 3rd generation cephalosporins (ceftriaxone) were given parenterally and 2nd generation cephalosporins (cefuroxime) were given both orally and parenterally (Table 3 ).

\section{Antibiotic use per source of infection}

The most common indications for antibiotic treatment were infections of the skin, soft tissue, bone and joints (SSTBJ) $(62.5 \%(140 / 224))$ and gastrointestinal tract (GIT) $(9.8 \%(22 / 224))$. For prophylaxis, 51.4\% (74/144)

Table 3 Antibiotic use pattern with respect to route of administration

\begin{tabular}{|c|c|c|}
\hline & \multicolumn{2}{|c|}{ Route of administration (Number of patients = $382(\%)$} \\
\hline & Oral $n=176(46.1 \%)$ & Parenteral $n=206(53.9 \%)$ \\
\hline Antibiotic group (n) & & $p$-value $=0.002$ \\
\hline Aminoglycosides (10) & 0 & $10(100.0)$ \\
\hline Penicillin (28) & $10(35.7)$ & $18(64.5)$ \\
\hline Cephalosporins (92) & $41(44.6)$ & $51(55.4)$ \\
\hline Carbapenems (5) & 0 & $5(100.0)$ \\
\hline$\beta$-lactam/ $\beta$-lactamase inhibitor (88) & $46(52.3)$ & $42(47.7)$ \\
\hline Lincosamides (51) & $28(54.9)$ & $23(45.1)$ \\
\hline Macrolides (1) & $1(50.0)$ & $1(50.0)$ \\
\hline Fluoroquinolones (53) & $30(56.6)$ & $23(43.4)$ \\
\hline Nitroimidazoles (51) & $17(33.3)$ & $34(66.7)$ \\
\hline Nitrofurantoin (2) & $2(100.0)$ & 0 \\
\hline Folate inhibitors (1) & $1(100.0)$ & 0 \\
\hline \multicolumn{3}{|l|}{ Duration of antibiotic intake / day } \\
\hline Median (range) & $6(0-89)$ & $4(1-33)$ \\
\hline$[\mathrm{IQR}]$ & [3-9] & {$[2-7]$} \\
\hline
\end{tabular}


and $25 \%(36 / 144)$ were given to prevent infections of SSTBJ and GIT infections, respectively. This picture was similar across hospital levels. Of note, cephalosporins (cefuroxime), $\beta$-lactam//lactamase inhibitors, clindamycin and metronidazole use dominated treatment of skin, soft tissue, bone and joint infections whereas fluoroquinolone and metronidazole dominated treatment of gastro-intestinal tract infections (Fig. 2).

\section{Duration of antibiotic use}

The median duration of antibiotic therapy prior to the survey was five days (IQR 3-8 days) with differences across different hospital levels $(p=0.02)$. It was 4 days (range 0-51 days, IQR 2-7 days) in district hospitals, with a wider range of 1-89 days (median 5 days, IQR: $3-8$ days) in tertiary level hospitals.

Depending on the indication, median duration of therapy varied from 6 days (IQR 3-9 days) for hospital acquired infections to 1 day for surgical prophylaxis given for one day, $(p=0.001)$.

In the surgical units, 65 HAIs were identified for $11 \%$ of patients (62/540). Of these, 56\% (35/62) were surgical site infections (35/62) and $95.2 \%(59 / 62)$ were on antibiotics.

About $4 \%(14 / 382)$ of patients on antibiotics had microbiological analysis done on at least one clinical sample: eight of these in three district hospitals, none of which had microbiological services, but in such cases sent samples to private laboratories, and six from one teaching hospital.

\section{Discussion}

This study shows a high prevalence of antibiotic use (70.7\%) among patients admitted to surgical wards across selected hospitals in Ghana. The study population represents almost a $1 / 3$ of the hospital capacity in Ghana

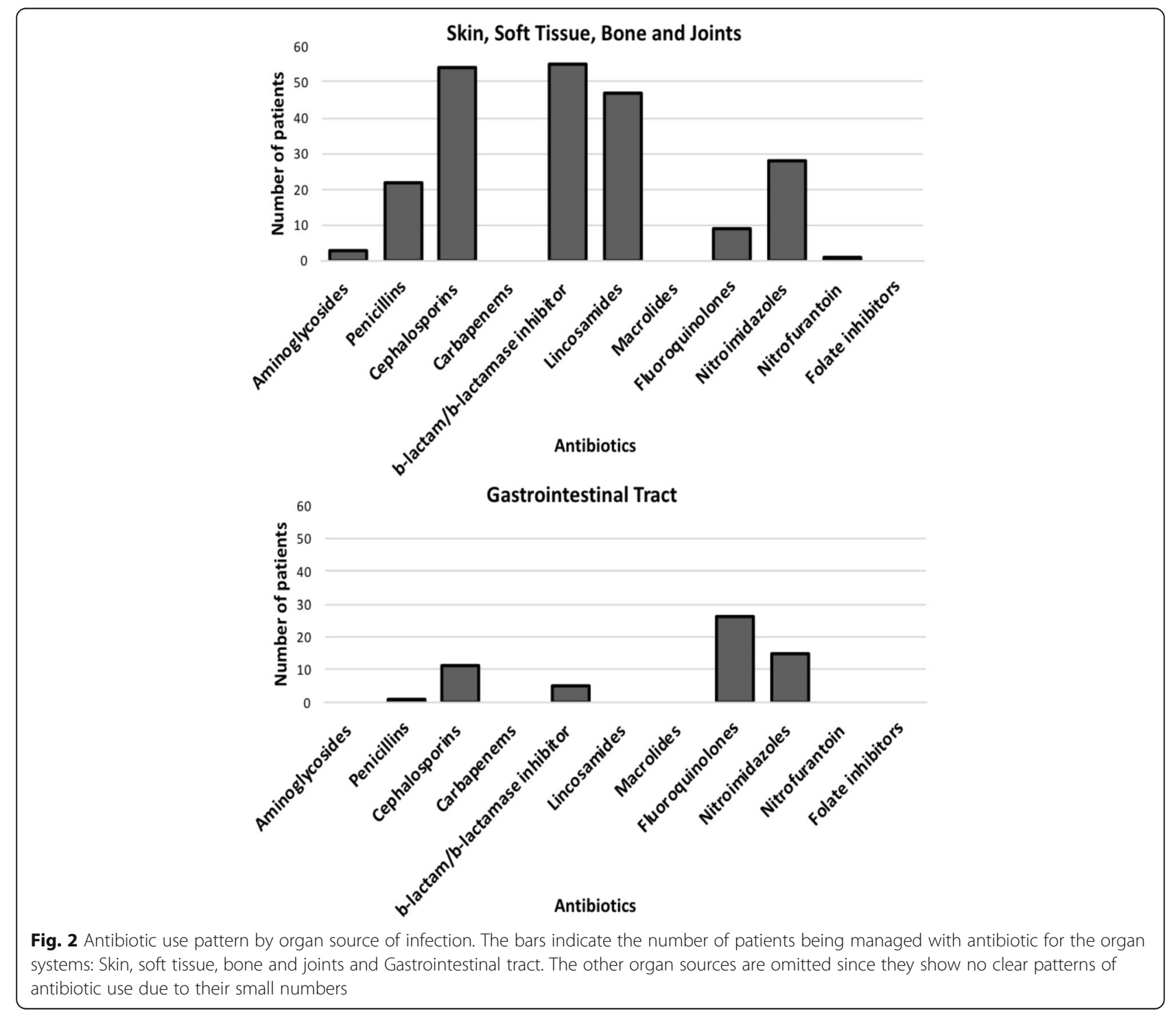


and is a fair representation of the surgical population across the country. Although teaching hospitals have a larger proportion of surgical patients due to their specialized personnel/ services and their overall bed size capacity, the results are likely to be representative of the country as a whole.

Antibiotic use was highest in patients admitted with urological conditions, and amongst patients who had undergone a surgical procedure or had developed a HAI. All the patients admitted with a urologic condition in this study were from a single tertiary hospital where there is a long waiting time for patients to see urologists and a longer time still for those requiring surgeries to have them done. Such patients may develop recurrent urinary tract infections requiring antibiotics or if surgery is performed on the urinary system, have an increased risk of developing SSIs. This may explain the higher use of antibiotics. There are low numbers of urologist in the country and lack of a urology center dedicated to managing such large numbers of patients.

Interestingly, the primary indication for antibiotic use was treatment of community-acquired infections, whereas only $1 / 3$ was used for surgical prophylaxis. This calls into question the usefulness of previous studies in surgical wards in LMIC that have mostly focused on surgical prophylaxis [16-19].

The rate of antibiotic use in this study is similar to the $70.8 \%$ recorded over a decade ago in a teaching hospital in Ethiopia [20] and higher than the 51\% recorded in a previous study looking at antibiotic use overall in a tertiary facility in Ghana [21]. The prevalence is higher than what is reported from the United States, 50\% [22] and in acute care hospitals in Europe, 36\% [15]. The high rate of antibiotic use brings to fore the absence of a national antibiotic use policy at the time of the study that would guide and control the use of antibiotics in Ghana [23]. The policy on antimicrobial use and resistance for Ghana [24] and the Ghana national action plan on antimicrobial resistance [25], both documents launched in 2018 by the Ministry of Health, Ghana, may be important to counteract the lack of antimicrobial resistance surveillance for both humans and animals [12]. The new guidelines may address, with their aim to improve infection control, pharmacovigilance and postmarketing surveillance of medications as well as expanding laboratory testing, the concern of high rates of antibiotic consumption demonstrated by this study.

There was a high use of 2nd and 3rd generation cephalosporins in community- and hospital-acquired infections as well as for medical and surgical prophylaxis. This goes against the recommendations in the standard treatment guideline of the Ministry of Health of Ghana to avoid cephalosporins to help reduce development of antibiotic resistance in the country [26]. In contrast, the guidelines recommend the use of penicillins (amoxicillin, ampicillin or cloxacillin) for community-acquired infections [26]. It is likely that clinicians wished to safeguard patients by using broader spectrum antibiotics [27]. In the absence of microbiological diagnosis, there was no possibility of scaling down to the appropriate antibiotic therapy based on results of culture and antibiotic susceptibility tests [28]. On average, 1.6 antibiotic agents were administered per patient. In one European study, 1.36 antibiotic agents are prescribed per patient on antibiotics [15]. Potentially, the number of antibiotics per prescription could also be reduced if microbiological analysis was carried out to find the most appropriate therapy. Even in the absence of microbiological testing at the individual level, regular microbiological surveys, including sensitivity testing, would be useful to inform treatment guidelines.

In this study, the most commonly used antimicrobials were metronidazole, cephalosporins, amoxicillin/clavulanic acid, fluoroquinolones and clindamycin, and similar agents were used for antibiotic therapy for communityand hospital-acquired infections as well as for surgical prophylaxis. Carbapenems were used at a low rate and only in tertiary hospitals. Our study design did not allow for us to directly judge the appropriateness of the choice of antibiotics. We did, however, notice that half of the antibiotics given for surgical prophylaxis were nitroimidazole and cefuroxime as recommended in the Ghana standard treatment guideline for use as surgical antibiotic prophylaxis, especially in the instances of colorectal or appendix surgery [26].

Most patients, having spent a median of nine days in hospital prior to the survey, would have had ample time for microbiological analysis to be carried out. The low rate of routine microbiological testing has been attributed to lack of diagnostic facilities [23, 29]. Regional and tertiary institutions in Ghana are all equipped with diagnostic facilities including the 10 study sites. However, only half of the study sites had facilities for microbiological analysis. The low rate of microbiological testing may not only be due to lack of microbiological testing facilities as seen in this study where district hospitals that did not have microbiological services used the services of private laboratories. Clinicians are key in the fight against antimicrobial resistance and their education on the need for microbiological testing whilst prescribing empirical antibiotics is important. Another reason for the low rate of microbiological testing may be that microbiological analyses are not covered by the National Health Insurance Scheme (NHIS) in Ghana. Thus, the patients will have to bear these costs [30]. The NHIS in Ghana, with an almost $40 \%$ coverage of the population [31], would have to review its cost covering policy to help reduce this low rate of microbiological testing. The 
lack of qualified microbiologists, unstable supplies of culture media and other essential reagents and equipment, as well as lack of tradition to do cultures may also account for the low rate of microbiological testing [32].

Previous antimicrobial treatment and excessive duration of treatment are the two most important factors in the selection of resistant microorganisms [33]. Though this study did not look at previous use of antibiotics in the admitted patients, it found that the median duration of administration of antibiotics on the survey date, across selected hospitals was five days, with some antibiotics administered for as long as 89 days. Surgical prophylaxis was generally given for long durations, i.e., more than the one day that is evidence-based and recommended by the World Health Organization [34]. Prolonged surgical prophylaxis does not reduce the number of wound infections and is rather associated with an increased risk of antimicrobial resistance and side effects [35].

Our study shows a widespread need for antibiotic stewardship programs, and for qualitative and quantitative monitoring and evaluation of healthcare professionals' attitudes toward antibiotic use and microbiological testing. It also suggests the need for improving microbiological services, to forestall the problem of inadequate antibiotic use [36-38], with clinical microbiologist playing a key role in antibiotic stewardship programs. Currently, there is limited evidence for the effect of antibiotic stewardship programs in LMIC. Our findings provide a baseline, urgently needed for studies that may provide such evidence.

\section{Limitations}

The data were collected based on a review of the health records, i.e., paper records of patients who were on admission on the survey day. The district and regional hospitals used similar booklets for everyday recording of patient management and teaching hospitals used similar folders. Because there was limited variation in the format of the records, both types of paper records were easy to review and deemed reliable for obtaining standard information on patient management.

The data on antibiotic use were limited, however, by the fact that this report only looks at the antibiotic use on the day of the survey and not all the antibiotics used by patients during the current admission, prior to the survey date. In addition, all indications were based on the judgement of the attending clinicians and were not scrutinized by the research team.

The timing of administration of surgical antibiotic prophylaxis was not assessed in the study.

The microbiological analysis may be underreported since only available microbiological reports were reviewed without respect to those which had been requested or those done and awaiting results, and this represents another limitation.

\section{Conclusion}

There is a high prevalence of antibiotic use in surgical practice at all levels of hospital care throughout Ghana. Our study elucidated problems on the appropriate use of antibiotics, which included long duration of antibiotic use and limited microbiological testing. For hospitalized patients there is strong evidence from high income countries of an effect of antibiotic stewardship programs on reduction of antibiotic use and resulting benefits at individual and societal levels. Our findings form the basis of and strongly call for studies in Ghana aiming at testing the effects of tailored antibiotic stewardship programs in LMIC. Policy guidelines on antibiotic use should be re-emphasized for all stake holders in Ghana.

\section{Additional file}

Additional file 1: Table S1: Combination of antibiotics prescribed in patients. This shows antibiotic combinations prescribed in patients. Each column is the antibiotic group added on to the initial column for the 382 patients. A row gives an indication of antibiotics combination for patients (number of patients on that antibiotic). NB. 169 patients were on 1 antibiotic, 178 patients on 2 antibiotics, 29 patients on 3 antibiotics and 6 patients on 4 antibiotics. (DOCX $18 \mathrm{~kb}$ )

\section{Abbreviations \\ BSI: Blood stream infection; CAl: Community acquired infection; \\ Cl: Confidence interval; ECDC: European Center for Disease Prevention and Control; GIT: Gastrointestinal tract; HAl: Healthcare associated infection; IQR: Interquartile range; KBTH: Korle-Bu Teaching Hospital; PPS: Point Prevalence Study; SP: Surgical prophylaxis; SSI: Surgical site infection; SSTBJ: Skin, soft tissue, bone and joints; UTI: Urinary tract infection; WHO: World Health Organization}

\section{Acknowledgements}

We thank all the members of the data collection team, management and staff of all the participating hospitals and Mr. Edmund Tetteh Nartey of the Centre for Tropical Clinical Pharmacology and Therapeutics, School of Medicine and Dentistry, University of Ghana, for his contribution during the database development.

Authors' contribution

$A B B, E O, A K L, N O N, G S M, S B, C B, J A O$ and MJN conceptualized the study; participated in its design, coordination, and questionnaire administration; and helped to draft the manuscript. ABB performed the statistical analysis. The manuscript was revised for intellectual content by KM, JALK and MJN. All authors have read and approved this manuscript.

\section{Funding}

The study was funded by DANIDA grant no. 16-PO1-GHA. The funding agency had no role in the design of the study, collection, analysis and interpretation of data, nor in writing the manuscript.

\section{Availability of data and materials}

The datasets used and/or analysed during the current study are available from the corresponding author on reasonable request.

\section{Ethics approval and consent to participate}

Ethical approval was granted by the Ethical Review Committees of the Ghana Health Service (GHS-ERC 08/05/2016) and the Institutional Review Board of KBTH (KBTH-STC/IRB/00044/2016). Individual informed consent was waived for the conduct of this study by the above ethics committees as it was deemed a surveillance activity. Anonymity was kept ensuring patient confidentiality. 


\section{Consent for publication}

Not applicable.

\section{Competing interests}

The authors declare that they have no competing interests.

\section{Author details}

'Department of Surgery, School of Medicine and Dentistry, College of Health Sciences, University of Ghana, Accra, Ghana. ${ }^{2}$ Department of Surgery, Korle Bu Teaching Hospital, Accra, Ghana. ${ }^{3}$ Department of Veterinary and Animal Science, University of Copenhagen, Copenhagen, Denmark. ${ }^{4}$ Division of Infectious Disease Preparedness, Statens Serum Institut, Copenhagen, Denmark. ${ }^{5}$ Department of Medical Laboratory Sciences, School of Biomedical and Allied Health Sciences, College of Health Sciences, University of Ghana, Accra, Ghana. ${ }^{6}$ Department of Microbiology, Korle Bu Teaching Hospital, P.O. Box 77, Accra, Ghana. ${ }^{7}$ Department of Clinical Microbiology, Copenhagen University Hospital (Righospitalet), Copenhagen, Denmark. ${ }^{8}$ Centre for Medical Parasitology, Department of Immunology and Microbiology, University of Copenhagen, Copenhagen, Denmark. ${ }^{9}$ Department of Medical Microbiology, School of Biomedical and Allied Health Sciences, College of Health Sciences, University of Ghana, Accra, Ghana. ${ }^{10}$ Department of Public Health, Global Health Section, University of Copenhagen, Copenhagen, Denmark. ${ }^{11}$ Formerly Institutional Care Division, Ghana Health Service, Accra, Ghana. ${ }^{12}$ Discipline of Community Health, Accra College of Medicine, P. O. Box CT 9828, Cantonments, Accra, Ghana.

Received: 2 November 2018 Accepted: 13 June 2019

Published online: 21 June 2019

\section{References}

1. WHO global strategy for containment of antimicrobial resistance, 2001.

2. Davey P, Marwick CA, Scott CL, Charani E, McNeil K, Brown E, et al. Interventions to improve antibiotic prescribing practices for hospital inpatients (review). Cochrane Database Sys Rev. 2017;(2) CD 003543.

3. Van Dijck C, Vlieghe E, Cox JA. Antibiotic stewardship interventions in hospitals in low- and middle-income countries: a systematic review. Bull World Health Organ. 2018:96:266-80.

4. Laxminarayan R, Duse A, Wattal C, Zaidi AKM, Wertheim HFL, Sumpradit N et al. Antibiotic resistance - the need for global solutions. Lancet Infect Dis. 2014;13:1057-98.

5. Saleh N, Awada S, Awwad R, Jibal S, Arfoul C, Zaiter L, et al. Evaluation of antibiotic prescription in the Lebanese community: a pilot study. Infect Ecol Epidemiol. 2015;5:27094.

6. Gharbi M, Moore LSP, Castro-Sánchez E, Spanoudaki E, Grady C, Holmes AH, et al. A needs assessment study for optimizing prescribing practice in secondary care junior doctors: the antibiotic prescribing education among doctors (APED). BMC Infect Dis. 2016;16(1):456.

7. Ingram P, Seet J, Budgeon C, Murray R. Point prevalence study of inappropriate antibiotic use at a tertiary Australian hospital. Int Med J. 2012; 42(6):719-21.

8. Ayukekbong JA, Ntemgwa M, Atabe AN. The threat of antimicrobial resistance in developing countries: causes and control strategies. Antimicrobial Resistance \& Infection Control. 2017;6:47.

9. Ashcroft DM, Lewis PJ, Tully MP, Farragher TM, Taylor D, Wass V, et al. Prevalence, nature, severity and risk factors for prescribing errors in hospital inpatients: prospective study in 20 UK hospitals. Drug Saf. 2015;38:833-43.

10. Slight SP, Howard R, Ghaleb M, Barber N, Franklin BD, Avery AJ. The causes of prescribing errors in English general practices: a qualitative study. $\mathrm{Br}$ J Gen Pract. 2013;63:e713-20.

11. Newman MJ, Frimpong E, Donkor ES, Opintan JO, Asamoah-Adu A. Resistance to antimicrobial drugs in Ghana. Infect and Drug Resist. 2011:4:215-20.

12. Yevutsey SK, Buabeng KO, Aikins M, Anto BP, Biritwum RB, Frimodt-Møller N, Gyansa-Lutterodt M. Situational analysis of antibiotic use and resistance in Ghana: policy and regulation. BMC Public Health. 2017;17:896-902.

13. Labi A-K, Obeng-Nkrumah N, Owusu E, Bjerrum S, Bediako-Bowan A, Sunkwa-Mills G, Akuffo C, Fenny JA, Enweronu-Laryea C, Debrah S, Damale N, Bannerman C, Newman MJ. Multi-center point prevalence survey of hospital-acquired infections in Ghana. J Hosp Infect. 2018. https://doi.org/10. 1016/j.jhin.2018.04.019.

14. Nsiah-Asare A. The health sector in Ghana: facts and figures 2017. Accra: Ghana Health Service; 2017.
15. ECDC. Point prevalence survey of healthcare-associated infections and antimicrobial use in European acute care hospitals. Stockholm: European Centre for Disease Prevention; 2013.

16. Mansour MA, Khalifa MO. Antibiotic prophylaxis in transarterial chemoembolization of hepatocellular carcinoma. Arab J Gastroenterol. 2018; 19(1):16-20.

17. Aiken AM, Wanyaro AK, Mwangi J, Juma F, Mugoya IK, Scott JAG. Changing use of surgical antibiotic prophylaxis in Thika hospital, Kenya: a quality improvement intervention with interrupted time series design. PLoS One. 2013;8(11):e78942

18. Dlamini LD, Sekikubo M, Tumukunde J, Kojjo C, Ocen D, Wabule A, et al. Antibiotic prophylaxis for caesarean section at a Ugandan hospital: a randomized clinical trial evaluating the effect of administration time on the incidence of postoperative infections. BMC Pregnancy Childbirth. 2015;15(1):1-7.

19. Suehiro T, Hirashita T, Araki S, Matsumata T, Tsutsumi S, Mochiki E, et al. Prolonged antibiotic prophylaxis longer than 24 hours does not decrease surgical site infection after elective gastric and colorectal surgery. Hepatogastroenterology. 2018;55(86-87):1636-9.

20. Abula T, Kedir M. The pattern of antibiotic usage in surgical in-patients of a teaching hospital, Northwest Ethiopia. Ethiop J Health Dev. 2004;18(1):35-8.

21. Labi A-K, Obeng-Nkrumah N, Nartey ET, Bjerrum S, Adu-Aryee NA, OforiAdjei YA, et al. Antibiotic use in a tertiary healthcare facility in Ghana: a point prevalence survey. Antimicrob Resist Infect Control. 2018 Jan 26;7:15.

22. Magill SS, Edwards JR, Beldavs ZG, et al. Prevalence of antimicrobial use in US acute care hospitals, may-September 2011. JAMA. 2014;312:1438-46.

23. Gyansa-Lutterodt M. Antibiotic resistance in Ghana. Lancet Infect Dis. 2013 Dec;13(12):1006-7.

24. Ministry of Health. Ghana National Action Plan for antimicrobial use and Resistance Republic of Ghana. 1st ed; 2017.

25. Ministry of Health. Republic of Ghana Policy on Antimicrobial Use and Resistance in ' One Health ' 1 st Edition, 2016 Ministry of Health. 2016.

26. Ministry of Health. Standard treatment guideline. Republic of Ghana. 6th ed; 2010

27. Om C, Daily F, Vlieghe E, JC ML, ML ML. 'If it's a broad spectrum, it can shoot better': inappropriate antibiotic prescribing in Cambodia. Anti- microb Resist Infect Control. 2016;5:58.

28. Sartelli M, Weber DG, Ruppe E, Bassetti M, Wright BJ, Ansaloni L, et al. Antimicrobials: a global alliance for optimizing their rational use in intraabdominal infections (AGORA). World J Emerg Surg. 2016;11:33.

29. Byarugaba DK. Antimicrobial resistance in developing countries and responsible risk factors. Int J Antimicrob Agents. 2004;24(2):105-10.

30. Kushitor MK, Boatema S. The double burden of disease and the challenge of health access. Evidence from access, bottlenecks, cost and equity facility survey in Ghana. PLoS One. 2018;13(3):1-11.

31. Fenny AP, Yates $R$, Thompson R. Social health insurance schemes in Africa leave out the poor. Int Health. 2018;10(1):1-3.

32. Polage CR, Bedu-Addo G, Owusu-Ofori A, Frimpong E, Lloyd W, Zurcher E, et al. Laboratory use in Ghana: physician perception and practice. Am J Trop Med Hyg. 2006;75(3):526-31.

33. Harbath S, Samore MH, Lichtenberg D, Cameli Y. Prolonged antibiotic prophylaxis after cardiovascular surgery and its effects on surgical site infections and antimicrobial resistance. Circulation. 2000;101:2916-21.

34. Global guidelines for the prevention of surgical site infections, 2016.

35. Suehiro T, Hirashita T, Araki S, Matsumata T, Tsutsumi S, Mochiki E, et al. Prolonged antibiotic prophylaxis longer than 24 hours does not decrease surgical site infections after elective gastric and colorectal surgery. Hepatogastroenterology. 2008;55(86-87):1636-9.

36. Barbé B, Yansouni CP, Affolabi D, Jacobs J. Implementation of quality management for clinical bacteriology in low-resource settings. Clin Microbiol Infect. 2017;23(7):426-33.

37. Sartelli M, Labricciosa FM, Barbadora P, Pagani L, Ansaloni L, Brink AJ, et al. The global Alliance for infections in surgery: defining a model for antimicrobial stewardship - results from an international cross-sectional survey. World J Emerg Surg. 2017;12:34.

38. Ombelet $\mathrm{S}$, Ronat J-B, Walsh T, Yansouni CP, Cox J, Vlieghe E, et al. Clinical bacteriology low-resource settings: today's solutions. Lancet Infect Dis. 2018; 18(8):e248-58.

\section{Publisher's Note}

Springer Nature remains neutral with regard to jurisdictional claims in published maps and institutional affiliations. 